\title{
Optimal Task Allocation Algorithm for Cost Minimization and Load Balancing of GSD Teams
}

\author{
Sundas Iftikhar, Anum Tariq, and Shoab Ahmed Khan
}

\begin{abstract}
In global software development partitioning of work into tasks and proper allocation of those tasks to sites are important factors to maximize the benefit of GSD and to cope with its challenges as well. This paper presents a mathematical model based on minimizing the cost and time and load balancing in global software development. It uses multi level clustering technique to reduce the inter-communication cost and optimally assign tasks to the sites. Primarily clustering is done for work division and secondly clustering is done at time of allocation of tasks. Task and site attributes are also considered during clustering. This model is implemented using MATLAB tool and task allocation example is used to compare its performance with other task allocation.
\end{abstract}

Index Terms-Global software development (GSD), task allocation, work assignment, distributive software development (DSD), clustering.

\section{INTRODUCTION}

From last decade global software development has become a common practice. There are many charms for developing software globally like availability of resources, cost, time but there come many challenges and risks in communication and co-ordination between geographically dispersed groups of people. Research reveals that all of the benefits of GSD are not attained as expected as there are many challenges and risks in GSD that work as barriers in success of the projects and make it more difficult to achieve expected benefits. Proper planning and management strategies can help to maximize the benefits and minimize the challenges occur in GSD .when making strategies, one of the important decision is how to distribute work on dispersed locations. A proper work distribution can minimize communication and co-ordination problems in GSD. Decision process of task allocation can be improved using different characteristics of site and tasks. Execution cost and intercommunication cost are two important characteristics that must be considered during task distribution and allocation decision process.

There is several task allocation methods like load balancing, integer programming, divide and conquer, grid computing [1] are reported in literature for distributed production and distributed systems and specifically for GSD techniques in literature are modification request [2], global studio Project [3], distributed Cocomo [4], Simulation model [5], Reference model for GSD [6], 24 hour software

Manuscript received August 28, 2014; revised October 31, 2014. This work was supported in part by the Department of Computer Engineering, College of EME, National University of Sciences and Technology (NUST).

The authors are with the Department of Computer Engineering, College of EME, National University of Sciences and Technology (NUST), H-12, Islamabad, Pakistan (e-mail: sundasiftikhar12@ce.ceme.edu.pk, anum_tariq11@yahoo.com,kshoab@yahoo.com). development model [7], TAMARI [8]. Still there is a need for appropriate and efficient work load sharing strategies to improve the performance of GSD teams. Using this load sharing strategy work tasks should be allocated to all sites. First load sharing decisions should be mad and then allocate work in such a manner that minimizes the cost of project.

Clustering techniques play an important role in load balancing and task distribution. In this paper we have used multilevel clustering for load balancing and optimal task allocation.

\section{A. Objective of Research}

The objective of research is to answer these two questions that can help to increase the success factor of a project in GSD. Nowadays basic agenda in GSD is how to maximize advantages and minimize the challenges and risk. One of the important decision that help to maximize advantages and minimizing risk and challenges is allocation of work task in GSD.

In a research on setting a research agenda for GSD [9], researchers concentrated on work division and its allocation on dispersed location by different criteria. In [10] author have shown that there are different methods and models proposed but still this issue needs to be addressed with different prospective.

So we worked on distribution and task allocation of the data based on clustering. We analyzed existing techniques for task allocation in GSD aspect and identified that cost and time are the major factors for increasingly adoption of GSD. So we have taken these two attributes in to account during clustering for task.

\section{B. Research Methodology}

GSD is a highly valuable but complex field. Development of projects can be seen as execution of a number of work tasks on different sites. Success factor of GSD projects highly depends on arrangement of tasks on the sites, when tasks are to be executed on a site and in what order execution of tasks is done? Task allocation should be done in such a way that inter task communication can be minimized and site properties must suit the task execution requirement. When we talk about GSD, a proper work distribution is the key factor in success of a project. Efficient allocation of divided work tasks to different sites to minimize the communication and co-ordination problems between GSD teams is another important aspect. So question addressed in this work are:

RQ1: How to distribute work?

RQ2: How to allocate tasks to different sites?

So we worked on distribution and task allocation of the data based on clustering. We analyzed existing techniques for task allocation in GSD aspect and identified that cost and time are the major factors for increasingly adoption of GSD. 
So we have taken these two attributes in to account during clustering for task.

\section{RESEARCH IDEA}

To answer the research questions we propose a multilevel clustering technique that optimally distributes work in to task clusters and assign these clusters to site that best suits the requirements of work tasks. Detail of technique is described in this section.

\section{A. Definitions and Equations}

Communication cost: Inter-task communication cost $\mathrm{CC}_{\mathrm{i}}$ is total cost needed for inter- communication between $\mathrm{T}_{\mathrm{i}}$ and $T_{j}$ residing on two different site. $\mathrm{CC}$ for all the tasks executing on one site is taken as zero.

Execution cost: The Execution $\operatorname{CostEC}_{\mathrm{ik}}$ is the total cost of executing a task $T_{i}$ on the site $S_{k}$. if the task $T_{i}$ is not executable on $S_{k}$ than execution cost is taken as infinite. Execution cost of a task $T_{i}$ on a site $S_{j}$ can be calculated as

$$
\sum_{i=1}^{n} \sum_{i=1}^{m} E C_{i k} X_{i k}
$$

whereas $X_{i k}=1$ only if task $I$ assigned to $k^{\text {th }}$ processor, zero otherwise.

Total cost: Total cost of project is equal to sum of total EC and ITCC.

\section{Total Cost=EC + ITCC}

Task clustering: Distance between every point and cluster mean can be calculated as

$$
\sum X_{i l c k} Z_{k i}\left\|X_{i}-\bar{X}_{k}\right\|^{2}=\sum_{i=1}^{m} Z_{k i}\left\|X_{i}-\bar{X}_{k}\right\|^{2}
$$

where mean can be calculated as

$$
X_{k}=1 / m_{k} \sum_{X i L C k} Z_{k i}
$$

$m_{k}$ is the total number of points assigned to that cluster

$$
m_{k}=\sum_{i=1}^{m} Z_{k i}
$$

$Z_{k i}$ is a suitability indicating parameter which gives indication of whether a cluster suits a data point. As sum of cluster compactness measure indicates the clustering goodness so over all goodness of cluster can be calculated as

$$
\varepsilon_{k}=\sum_{i=1}^{m} \sum_{k=1}^{k n} Z_{k i}\left\|X_{i}-\bar{X}_{k}\right\|^{2}
$$

Find value of $X_{K}$ such that $\varepsilon_{k}$ can be minimized.

\section{B. Technique}

We considered a GSD environment where there are $m$ number of tasks $T=\{T 1, T 2, T 3 \ldots \ldots . . T m\}$ which are to be allocated on n number of sites $S=\{S 1, S 2, S 3 \ldots \ldots . S n\}$. The execution cost and communication cost are known and mentioned in ECM and ITCCM respectively. Since the major goal is optimal task allocation to geographically dispersed sites to minimize the cost of overall project, so tasks will same attributes will be grouped together. Number of cluster will be equal to number of sites included. On this level $k$-mean clustering will group task in to $k$ cluster. Here m vectors of tasks are to be placed onto k clusters. For grouping the $\mathrm{m}$ vectors, $k$ centroids are calculated to make $\mathrm{k}$ clusters. For all task of Execution cost matrix, assign each task vector to the nearest centroids. This iteration of assignment and recalculation will continue till centroids stop moving. As a result of this process we will get clusters from which we will calculate the matrices using equation (3).

For all the clusters add execution time of the tasks grouped in one cluster in ECM matrix, and modify ITCCM matrix by putting communication cost for the task laying in same cluster which is equal to zero.

For assigning clusters of task to $\mathrm{n}$ sites se will do clustering on basis of $\mathrm{EC}_{\mathrm{ik}}$ be done on the basis of ecil (execution cost) constraint. Modify ECM and ITCCM accordingly. Once task are assigned, optimal assignment cost and total cost are calculated using equation (2)

\section{Assumptions}

1) Work is assumed to be collection of $m$ tasks which are to be allocated on $n$ sites.

2) All site have different attributes.

3) Total no. of tasks to be assigned is more than the no. of site $(m>>n)$.

4) Inter-communication cost between two tasks executing on one site is assumed to be zero. Irrespective from time, when a task cluster is assigned to a site the ITCC will be zero within that cluster.

5) If a task is not executable on a site than its execution cost for that site is taken infinite.

6) Collection of EC vectors (which represent execution cost of one task on each site) will be shown with data points of $k$-mean clustering

\section{Notations}

TABLE I: NOTATIONS

\begin{tabular}{|l|l|}
\hline Notation & Description \\
\hline$T$ & Set of tasks \\
\hline$S$ & Set of sites involved in development \\
\hline$M$ & Number of sites \\
\hline$N$ & Number of task \\
\hline$T_{i}$ & $i^{\text {th }}$ task of givenset \\
\hline$S_{k}$ & $K^{\text {th }}$ site in $S$ \\
\hline$X$ & $\begin{array}{l}\text { allocation matrix }, X_{i k}=1 \quad \text { assignment of } i^{\text {th }} \text { task } \text { to } K^{\text {th }} \\
\text { site } \text { in } 0 \text { otherwise }\end{array}$ \\
\hline$E C$ & Execution cost \\
\hline$C C$ & communication cost \\
\hline$E C M$ & execution cost matrix \\
\hline$I T C C M$ & inter task communication cost matrix \\
\hline$C L$ & cluster information vector \\
\hline
\end{tabular}

Description of notations used in Algorithm is given in Table I.

\section{E. Proposed Algorithm}

1) Read number of sites in $S$.

2) Read number of tasks in $T$. 
3) Read number of clusters in $k$.

4) Read ECM of order $m \times n$.

5) Read inter-task communication cost matrix (ITCCM).

6) Apply $k$ mean clustering on ECM

7) Store cluster information in vector CL.

8) Modify ECM by inserting execution time of tasks in each cluster.

9) Put inter-communication cost equal zero between the tasks which are on same cluster in ITCCM.

10) Divide modified ECM in $n$ column matrix, which includes execution cost of each work task on site $S_{i}$.

11) Sort each column matrix

12) For each column matrix

$I=1$ to $n$

For $j=1$ to $m$

If ( $T_{j}$ is not assigned)

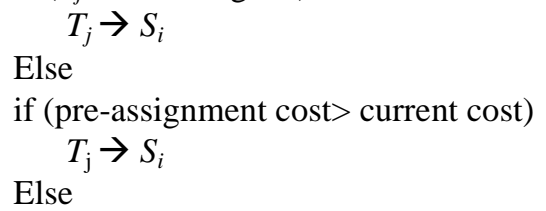

skip current assignment

End if

End if

End for

End for

13) Calculate total EC and ITCC

14) Optimal cost $=\mathrm{EC}+$ ITCC

End

\section{IMPLEMENTATION}

We considered a GSD environment having nine tasks $T 1$, $T 2, T 3, T 4, T 5, T 6, T 7, T 8, T 9$ which are to be allocated on three sites $S 1, S 2, S 3$. As number of sites is three so we will take number of clusters equal to the number of sites.

Number of cluster $=3$

Value of ECM and ITCCM are given in Table II and Table III respectively.

TABLE II: EXECUTION COST METRIX (ECM)
\begin{tabular}{|l|l|l|l|}
\hline & $S 1$ & $S 2$ & $S 3$ \\
\hline$T 1$ & 174 & 176 & 110 \\
\hline$T 2$ & 95 & 15 & 134 \\
\hline$T 3$ & 196 & 79 & 156 \\
\hline$T 4$ & 148 & 215 & 143 \\
\hline$T 5$ & 44 & 234 & 122 \\
\hline$T 6$ & 241 & 225 & 27 \\
\hline$T 7$ & 12 & 28 & 192 \\
\hline$T 8$ & 215 & 13 & 122 \\
\hline$T 9$ & 211 & 11 & 208 \\
\hline
\end{tabular}

TABLE III: INTER-TASK COMMUNICATION COST METRIX (ITCCM)

\begin{tabular}{|c|c|c|c|c|c|c|c|c|c|}
\hline & $T 1$ & $T 2$ & $T 3$ & $T 4$ & $T 5$ & $T 6$ & $T 7$ & $T 8$ & $T 9$ \\
\hline$T 1$ & 0 & 8 & 10 & 4 & 0 & 3 & 4 & 0 & 0 \\
\hline$T 2$ & 8 & 0 & 7 & 0 & 0 & 0 & 0 & 3 & 0 \\
\hline$T 3$ & 10 & 7 & 0 & 1 & 0 & 0 & 0 & 0 & 0 \\
\hline$T 4$ & 4 & 0 & 1 & 0 & 6 & 0 & 0 & 8 & 0 \\
\hline$T 5$ & 0 & 0 & 0 & 6 & 0 & 0 & 0 & 12 & 0 \\
\hline$T 6$ & 3 & 0 & 0 & 0 & 0 & 0 & 0 & 0 & 12 \\
\hline$T 7$ & 4 & 0 & 0 & 0 & 0 & 0 & 0 & 3 & 10 \\
\hline 78 & 0 & 3 & 0 & 8 & 12 & 0 & 3 & 0 & 5 \\
\hline$T 9$ & 0 & 0 & 0 & 0 & 0 & 12 & 10 & 5 & 0 \\
\hline
\end{tabular}

On applying $\mathrm{K}$ mean clustering on ECM we get clusters $C 1=T 5, T 7$

$C 2=T 2, T 3, T 8, T 9$

$C 3=T 1, T 4, T 6$

Putting execution cost in each cluster as shown in Table IV.

TABLE IV: MODIFIED ECM AFTER 1ST LEVEL CLUSTERING

\begin{tabular}{|l|l|l|l|l|}
\hline & $S 1$ & $S 2$ & $S 3$ & \\
\hline$T 1$ & 174 & 176 & 110 & $\mathrm{C} 3$ \\
\hline$T 2$ & 95 & 15 & 134 & $\mathrm{C} 2$ \\
\hline$T 3$ & 196 & 79 & 156 & $\mathrm{C} 2$ \\
\hline$T 4$ & 148 & 215 & 143 & $\mathrm{C} 3$ \\
\hline$T 5$ & 44 & 234 & 122 & $\mathrm{C} 1$ \\
\hline$T 6$ & 241 & 225 & 27 & $\mathrm{C} 3$ \\
\hline$T 7$ & 12 & 28 & 192 & $\mathrm{C} 1$ \\
\hline$T 8$ & 215 & 13 & 122 & $\mathrm{C} 2$ \\
\hline$T 9$ & 211 & 11 & 208 & $\mathrm{C} 2$ \\
\hline
\end{tabular}

Putting ITCC equal zero between tasks which are residing on same cluster we get modified ITCCM given in Table V.

\begin{tabular}{|r|r|r|r|r|r|r|r|r|r|}
\multicolumn{10}{|c|}{ TABLE V: MODIFIED ITCCM } \\
\hline & $T 1$ & $T 2$ & $T 3$ & $T 4$ & $T 5$ & $T 6$ & $T 7$ & $T 8$ & $T 9$ \\
\hline$T 1$ & 0 & 8 & 10 & 4 & 0 & 3 & 4 & 0 & 0 \\
\hline$T 2$ & 8 & 0 & 0 & 0 & 0 & 0 & 0 & 0 & 0 \\
\hline$T 3$ & 10 & 0 & 0 & 1 & 0 & 0 & 0 & 0 & 0 \\
\hline$T 4$ & 4 & 0 & 1 & 0 & 6 & 0 & 0 & 8 & 0 \\
\hline$T 5$ & 0 & 0 & 0 & 6 & 0 & 0 & 0 & 12 & 0 \\
\hline$T 6$ & 3 & 0 & 0 & 0 & 0 & 0 & 0 & 0 & 12 \\
\hline$T 7$ & 4 & 0 & 0 & 0 & 0 & 0 & 0 & 3 & 10 \\
\hline$T 8$ & 0 & 0 & 0 & 8 & 12 & 0 & 3 & 0 & 0 \\
\hline$T 9$ & 0 & 0 & 0 & 0 & 0 & 12 & 10 & 0 & 0 \\
\hline
\end{tabular}

Then we divided modified ECM in n column metrics and again applied clustering algorithm on it as mentioned in step 12.using clustering again, we assigned the tasks in clusters to sites Resulting arrangement of tasks to sites is as below:

$$
T 5, T 7 \rightarrow S 1
$$

$$
\begin{gathered}
T 2, T 3, T 8, T 9 \rightarrow S 2 \\
T 1, T 4, T 6 \rightarrow S 3
\end{gathered}
$$

Calculated total execution cost from modified ECM given in Table VI.

TABLE VI: EXECUTION COST AT SITES

\begin{tabular}{|l|l|l|}
\hline tasks & sites & EC \\
\hline$T 5, T 7$ & $S 1$ & 56 \\
\hline$T 2, T 3, T 8, T 9$ & $S 2$ & 118 \\
\hline$T 1, T 4, T 6$ & $S 3$ & 280 \\
\hline total & & 454 \\
\hline
\end{tabular}

Calculated total inter-task communication cost from modified ITCCM as given in Table VII.

Using the total EC and ITCC calculate total cost of the system using equation (2).

So total system cost is $454+142=596$. 
TABLE VII: TOTAL INTER-TASK COMMUNICATION COST

\begin{tabular}{|c|c|c|}
\hline Sites & Tasks & ITCCM \\
\hline \multirow[b]{2}{*}{$S 1$} & $T 5$ & 18 \\
\hline & $T 7$ & 17 \\
\hline \multicolumn{2}{|l|}{ total } & 35 \\
\hline \multirow[b]{4}{*}{$S 2$} & $T 2$ & 8 \\
\hline & $T 3$ & 11 \\
\hline & $T 8$ & 23 \\
\hline & $T 9$ & 22 \\
\hline \multicolumn{2}{|l|}{ total } & 64 \\
\hline \multirow[b]{3}{*}{$S 3$} & $T 1$ & 22 \\
\hline & $T 4$ & 9 \\
\hline & $T 6$ & 12 \\
\hline \multicolumn{2}{|l|}{ total } & 43 \\
\hline Total ITCCM & & 142 \\
\hline
\end{tabular}

\section{COMPARISON OF RESUlTS}

To compare efficiency of proposed algorithm we used an example from [11], executed the proposed algorithm and compared its results with Bora et al. [11], Lo's et al. [12], and Kopidakis et al. [13]. Results are shown in Table VIII.

\begin{tabular}{|c|c|c|c|c|}
\hline \multicolumn{1}{|c|}{ TABLE VIII: COMPARISON RESULTS } \\
algorithm of & $\begin{array}{c}\text { Bora } \text { et al. } \\
\text { algorithm }\end{array}$ & $\begin{array}{c}\text { Lo's } \text { et al. } \\
\text { algorithm }\end{array}$ & $\begin{array}{c}\text { Kopidakis } \\
\text { et al. } \\
\text { algorithm }\end{array}$ & $\begin{array}{c}\text { Proposed } \\
\text { algorithm }\end{array}$ \\
\hline cost & 270 & 275 & 285 & 195 \\
\hline $\begin{array}{l}\text { Minimization in } \\
\text { cost w.r.t } \\
\text { proposed algo }\end{array}$ & $27.78 \%$, & $29.09 \%$ & $31.58 \%$ & \\
\hline
\end{tabular}

\section{CONCLUSIONS}

Cost is considered as a major factor for adopting GSD. Proposed algorithm in this paper considered static load sharing scheme for task allocation on globally distributed sites. It uses multi level clustering technique to reduce the inter-communication cost and optimally assign tasks to the sites In this work we have considered two task attributes execution cost and intercommunication task in future research we will focus on more attributes related to tasks and sites.

\section{REFERENCES}

[1] K. Govil, "A smart algorithm for dynamic task allocation for distributed processing environment," International Journal of Computer Applications, vol. 28, no. 2, pp.13-19, 2011.

[2] A. Mockus and D. M. Weiss, "Globalization by chunking: A quantitative approach," IEEE Software, vol. 18, issue 2. pp. 30-37, March 2001.

[3] M. Neel et al., "Siemens global studio project: Experiences adopting an integrated GSD infrastructure," in Proc. International Conference Global Software Engineering, pp. 203-212, Florianopolis, October 2006.
[4] M. Ray, "Distributed global development parametric cost modeling," in Proc. International Conference on Software Process, Dynamics and Agility, pp. 159-168, USA, Springer Berlin Heidelberg, 2007.

[5] S. Setamanit and D. Raffo, "Identifying key success factors for globally distributed software development project using simulation: A case study," in Proc. International Conference on Software Process, ICSP, pp. 330-332, Heidelberg, Germany: Springer, 2008.

[6] R. Prikladnicki, J. L. N. Audy, and R. Evaristo, "A reference model for global software development: Findings from a case study," in Proc. International Conference on Global Software Engineering ICGSE 2006, pp. 18-28, Florianopolis, October 2006.

[7] J. Pankaj and G. Jain, "Assigning tasks in a 24-hour software development model," Journal of Systems and Software, vol. 79, no. 7 . pp. 904-911, 2006.

[8] A. Lamersdorf and J. Münch, "TAMRI: A tool for supporting task distribution in global software development projects," in Proc. 2009 Fourth IEEE International Conference on Global Software Engineering, pp. 322-327, Limerick, July 2009.

[9] A. Lamersdorf and J. Münch, "A multi-criteria distribution model for global software development projects," Journal of the Brazilian Computer Society, vol. 16, no. 2, pp. 97-115, 2010.

[10] P. Yadav, M. Singh, and K. Sharma, "An optimal task allocation model for system cost analysis in heterogeneous distributed computing systems: A heuristic approach," International Journal of Computer Applications, vol. 28, no. 4, pp. 30-37, 2011.

[11] L. V. Mary, "Heuristic algorithms for task assignment in distributed system," IEEE Trans. Computer., vol. 37, issue 11, pp. 1384-1397, Nov. 1988.

[12] B. Ucara, C. Aykanat, K. Kayaa, and M. Ikincib, "Task assignment in heterogeneous computing system," Journal of parallel and Distributed Computing, vol. 66, no. 1, pp. 32-46, 2006.

[13] Y. Kopidakis, M. Lamari, and V. Zissimopoulos, "Task assignment problem: Two new heuristic algorithms," J. Parallel Distributive Computing, vol. 42, issue 1, pp. 21-29, 1997.

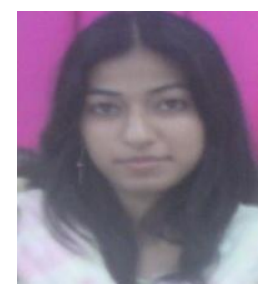

Sundas Iftikhar is from Pakistan. She completed her BSc in software engineering from Azad Jammu and Kashmir University, Pakistan in 2011 and now she is doing her M.Sc. in software engineering from National University of Science and technology (NUST) Pakistan from 2013. Her research interests include global software development and cloud computing.

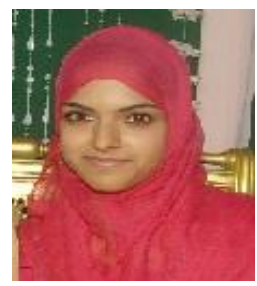

Anum Tariq is from Pakistan. She completed her BSc in software engineering from Azad Jammu and Kashmir University, Pakistan in 2010 and now she is doing her M.Sc. in software engineering from National University of Science and technology (NUST) Pakistan from 2013. Her research interests include cloud computing, global software development.

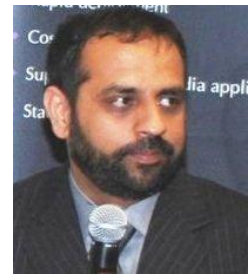

Shoab Ahmed Khan is from Pakistan. He is an associate professor at the Department Computer Engineering, National University of Science and technology (NUST) Pakistan, specialized in electrical and computer engineering, MSc /Ph.D. computer engineering from USA. His research interests include image processing, parallel processing, microcontroller system design, microprocessor design. 